\title{
First considerations on the SARS-CoV-2 epidemic in the Dialysis Units of Piedmont and Aosta Valley, Northern Italy
}

\author{
Marco Manganaro ${ }^{1}$ Simone Baldovino $0^{2,3}$ on behalf of The Working group of the Piedmont and Aosta Valley \\ Section of the SIN
}

Published online: 10 April 2020

(C) Italian Society of Nephrology 2020

Piedmont (4,356,406 inhabitants) and Aosta Valley (125,666 inhabitants [1]) are two Regions of North-Western Italy, covering an area of almost $29,000 \mathrm{~km}^{2}$ where 5793 patients are presently receiving renal replacement therapy (RRT): 2893 are on haemodialysis, 387 on peritoneal dialysis and 2513 have a functioning kidney graft [2].

In just over a month since the first case of SARS-CoV-2 positivity was recorded in Piedmont on February 22nd, 7782 $(0.17 \%)$ inhabitants in these two Regions have been infected, and 637 of them have died (lethality of 8.19\%) [3].

Since the outbreak of the epidemic nephrologists identified dialysis and kidney transplanted patients as a category at greater risk of contracting the infection and suffering its lethal consequences [4]. They also realized that the general health rules issued by the authorities were not sufficient to protect the nephrology-dialysis community. Nephrologists' warnings went initially unheeded. The main difficulties they had to deal with while trying to set up the most appropriate solutions included:

- regulating transport from home and access to the hospital, in particular for febrile patients, organizing telephone

The members of the Working group of the Piedmont and Aosta Valley Section of the SIN are listed in "Acknowledgements" section.

Marco Manganaro

mmanganaro@ospedale.al.it

1 Nephrology and Dialysis Unit, Azienda Ospedaliera "SS. Antonio e Biagio e Cesare Arrigo", Via Venezia 16, 15121 Alessandria, Italy

2 CMID-Nephrology and Dialysis Unit (ERK-Net Member), ASL Città di Torino - Hub S. Giovanni Bosco Hospital, Turin, Italy

3 Department of Clinical and Biological Sciences, Center of Research of Immunopathology and Rare Diseases-Coordinating Center of Piedmont and Aosta Valley Network for Rare Diseases, University of Turin, Turin, Italy pre-triage in order to arrange individual transport of suspected SARS-CoV-2 cases and to avoid group transportation,

- triaging patients before allowing them to enter the dialysis rooms,

- identifying designated areas where suspected and confirmed SARS-CoV-2 patients could undergo dialysis,

- guaranteeing adequate levels of protection (including personal protective equipment) for health care professionals, especially in designated areas, as well as a suitable number of surgical masks for the patients,

- broadening the indications for, and quickly getting the results of, nasopharyngeal and oropharyngeal swabs in individuals in contact with suspected or confirmed SARS-CoV-2 cases.

This was of paramount importance when dealing with such a tightly knit community of operators and patients continuously interacting with each other.

In a context in which data on dialysis and kidney transplant patients are few and scattered, we report our first experience concerning patients and professionals in the 22 Nephrology and Dialysis Units in Piedmont and Aosta Valley. The two Regions employ about 1200 health care workers including 226 nephrologists and 959 non-medical staff. The present report is updated 35 days after the beginning of the epidemic (March 27, 2020).

Data were updated in real time by all the Units via a IT platform developed by the local Nephrology Network and the Network of Rare Diseases of Piedmont and Aosta Valley, and were analyzed using SPSS Statistics version 26 (IBM, Armonk, USA), and Wizard version 1.9.40 (Evan Miller, Chicago, IL, USA).

No SARS-COV-2 cases were observed in the only Paediatric Nephrology Unit in the Region (having in charge 4 hemodialysis, 4 peritoneal dialysis, and 35 transplanted 
patients), which was not surprising due to the lower susceptibility of children to clinically evident infection.

In Piedmont and Aosta Valley, mainly symptomatic patients or patients at high risk of exposure (e.g. health care workers exposed to positive patients without adequate protection) have been tested until now. This approach may have caused a substantial underestimation of positive patients, preventing exact calculation of mortality rates. However, in the last days, the health authorities have extended indications, at least among healthcare professionals. Moreover, clinicians of the emergency and intensive care units are commonly using radiographic signs suggestive of interstitial pneumonia as surrogate criteria of SARS-CoV-2 disease.

In adults, 156 SARS-COV-2 cases were recorded: 130 RRT patients including 98 in hemodialysis, 4 in peritoneal dialysis, 26 transplanted, and 2 waiting for transplantation, and 26 health care workers ( 6 physicians, 18 nurses and 2 members of auxiliary staff). SARS-COV-2 cases were unevenly distributed within the Regions ranging from 0 to maximum of 25 cases per Nephrology Unit. The difference in number of cases among the Units may reflect a true variability in the incidence of positive cases in Piedmont and Aosta Valley. However, the high number of cases in single Centers might also be due to intra-hospital spreading of the infection. In this context, it is worth emphasising the need to comply with the correct hygienic rules, including hand disinfection and use of surgical masks by personnel and patients.

The incidence of confirmed cases showed an increasing trend between March 16th to 26th, slowing down in the last week, possibly due to the very restrictive measures adopted by the Italian government (Fig. 1).

The prevalence of SARS-CoV-2 positivity was the same in RRT patients and nephrology staff: $2.2 \%$. This is much higher than the rates for the general population in Piedmont and Aosta Valley (7782 subjects affected by SARS-CoV-2, i.e., $0.17 \%$ of the population), with an odds ratio of 13.43 , 95\% CI 11.27 to 16.00 ; $\mathrm{z}$ score $\mathrm{p}<0.0001$ for RRT patients,

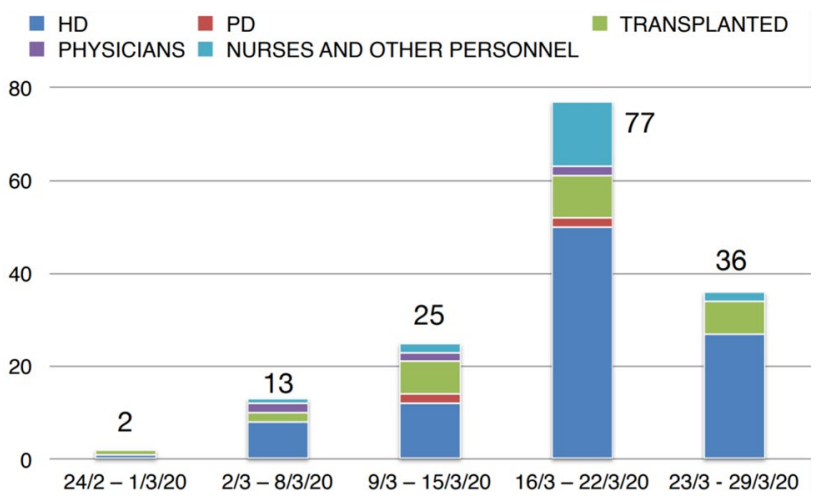

Fig. 1 Number of new confirmed (positive swab) cases per week and of 12.80 (95\% CI 8.67 to 18.89 ; $\mathrm{z}$ score $\mathrm{p}<0.0001)$ for staff.

Presently, all personnel affected by SARS-CoV-2 have been quarantined at home (waiting for two negative swabs at $24 \mathrm{~h}$-intervals to be allowed to come back to work), but one nurse who has been hospitalized.

The average age of affected patients was 69.7 (range 26-92 years). It was higher in dialysis (70 years, range 41-92) than in transplanted patients (61, range 26-80), with a higher number of males (59.3 vs $40.7 \%$ ), which is consistent, at least in part, with the prevalence of males in the RRT population.

Of these patients, $74.6 \%$ have been hospitalised. Being under RRT did not preclude per se admission to the intensive care unit (ICU). However, the incidence of ICU admission was higher in transplanted than dialysis patients, presumably due to lower comorbidity burden that favoured accessibility to intensive care. Anyway, the difference was not statistically significant $(20.8 \%$ vs $8.2 \%$; $z$-score $p=0.086)$. The death rate was $24.6 \%$, much higher than that observed in the whole SARS-CoV-2-positive population of the same area in the same period $(8.19 \%$; 637 deaths in 7,782 confirmed subjects affected by SARS-CoV-2). The Odds Ratio for death in RRT patients $v s$ the general population was 3.80 (95\% CI: 2.53 to 5.72; $\mathrm{z}$ score, $\mathrm{p}<0.0001$ ).

Lethality was higher in males than in females $(31.1 \%$ vs $4 \%$, respectively; $\mathrm{z}$ score, $\mathrm{p}=0.008$ ).

Lethality was not related to dialysis vintage, while the presence of cardiovascular diseases was associated with higher lethality $(29.9 \%$ vs $10.7 \%$; $=0.040)$. Diabetes was a risk factor in transplanted patients $(66.7 \%$ vs $13.6 \%$; $\mathrm{p}=0.031$ ). History of neoplasia was also associated with an increase in risk, albeit not statistically significant (40\% vs 19\%). Treatment almost invariably included anti-retroviral agents and hydroxychloroquine. The database was not designed to provide data on rescue therapies, such as anticytokine treatments. Deaths were due to multi-organ failure and cardiovascular collapse.

In summary, these preliminary data must be interpreted with caution and require further in-depth re-evaluation after the individual follow-up of each patient is completed. However, we felt that they could add to the current scant knowledge since they come from the first regional inquiry in RRT patients and may help better understanding of the epidemic. Furthermore, these data confirm that RRT patients and hospital staff have a higher incidence of infection and show that RRT patients have higher lethality than the general population. This observation may support maximizing preventive measures and early diagnosis in this fragile population.

Working group of the Piedmont and Aosta Valley Section of the SIN - all the Authors contributed equally to this work. Luca Besso-Nephrology and Dialysis Unit, S. Croce e Carle Hospital, Cuneo, Luigi Biancone-Renal Transplantation 
Center, "A. Vercellone", Division of Nephrology Dialysis and Transplantation, Città della Salute e della Scienza Hospital and Department of Medical Sciences, University of Turin, Turin, Italy, Roberto Boero- Nephrology and Dialysis Unit, Martini Nuovo Hospital, Turin, Maurizio BorzumatiNephrology and Dialysis Unit ASL VCO, Verbania, Giovanni Calabrese-Nephrology and Dialysis Unit ASL AL, Casale Monferrato-Novi Ligure, Vincenzo CantaluppiNephrology and Transplantation Unit, AOU Maggiore della Carità, Novara, Fabio Chiappero-Nephrology and Dialysis Unit, ASL TO3, Rivoli-Pinerolo, Doriana ChiarinottiNephrology and Dialysis Unit, AOU Maggiore della Carità, Novara, Stefano Cusinato-Nephrology and Dialysis Unit, ASL NO, Borgomanero, Oliviero Filiberti-Nephrology and Dialysis Unit, ASL VC, Vercelli, Marco Formica- Nephrology and Dialysis Unit, CN1, Savigliano-Saluzzo-MondovìCeva, Bruno Gianoglio-Pediatric Nephrology and Dialysis Unit AOU Città della Salute e della Scienza, Turin, Stefano Maffei-Nephrology and Dialysis Unit, ASL AT, Asti, Massimo Manes-Nephrology and Dialysis Unit, AUSL Aosta, Dario Roccatello-CMID-Nephrology and Dialysis Unit (ERK-net member), S. Giovanni Bosco Hospital and Department of Clinical and Biological Sciences-University of Turin, and Center of Research of Immunopathology and Rare Diseases-Coordinating Center of Piedmont and Aosta Valley Network for Rare Diseases, Colombano SaccoNephrology and Dialysis Unit, ASL BI, Biella, Mario Salomone-Nephrology and Dialysis Unit, ASL TO5, Chieri, Silvana Savoldi-Nephrology and Dialysis Unit ASL TO4, Cirié-Ivrea-Chivasso, Giuliana Tognarelli-Nephrology and Dialysis Unit AOU S. Luigi Gonzaga, Orbassano, Giusto Viglino-Nephrology and Dialysis Unit, ASL CN2, Alba, Corrado Vitale-Nephrology and Dialysis Unit AO Ordine Mauriziano, Turin, Antonio Amoroso and Silvia VanzinoImmunogenetic and Transplant Biology Center, Città della
Salute e della Scienza Hospital and Department of Medical Sciences, University of Turin, Turin, Italy.

\section{Compliance with ethical standards}

Conflict of interest All the authors declare that they have no conflicts of interest.

Ethical approval All data used in this study were collected in accordance with the ethical standards of the institutional and regional research committees, with the 1964 Helsinki Declaration and its later amendments or comparable ethical standards. According to the European GDPR, all data were anonymized before the collection.

\section{References}

ISTAT (2019) Italy, region, district resident population at January 1st, 2019 (online). https://dati.istat.it/Index.aspx?QueryId=18540. Accessed 29 Mar 2020

Maffei S, Maspoli M, Simonetti MT, Biancone L, Cusinato S, Forneris G, Tognarelli G, Manganaro M (2019) CKD regional observatory report 2018 (online). https://www.trapiantipiemonte.it/pdf/Resoc onti/ResocontoOMRC2018.pdf. Accessed 29 Mar 2020

Italian Ministry of Health (2020) Covid-19 Italian cases at 27 March 2020 (online). www.salute.gov.it/imgs/C_17_pagineAree _5351_40_file.pdf. Accessed 27 Mar 2020

Rombolà G, Heidempergher M, Pedrini L, Farina M, Aucella F, Messa P, Brunori G (2020) Practical indications for the prevention and management of SARS-CoV-2 in ambulatory dialysis patients: lessons from the first phase of the epidemics in Lombardy. J Nephrol. https://doi.org/10.1007/s40620-020-00727-y (published online ahead of print, 2020 Mar 23)

Publisher's Note Springer Nature remains neutral with regard to jurisdictional claims in published maps and institutional affiliations. 